\title{
Accesibilidad física en pistas polideportivas cubiertas de Extremadura: Un estudio exploratorio Physical accessibility at indoor sport courts in Extremadura: An exploratory study
}

\author{
Vicente Luis del Campo, Carlos Grande Claver, Alberto Moriano Hernández
}

Universidad de Extremadura (España)

\begin{abstract}
Resumen. El objetivo del estudio fue conocer el porcentaje medio de accesibilidad física de un conjunto de instalaciones deportivas convencionales en Extremadura, así como determinar el porcentaje de accesibilidad de cada de una de las zonas que formaban parte de dichas instalaciones deportivas. Específicamente, 38 pistas polideportivas cubiertas fueron analizadas (19 de la provincia de Cáceres y 19 de la provincia de Badajoz), cumplimentando un total de 1673 ítems con el cuestionario de accesibilidad propuesto por el Consejo Superior de Deportes y el Instituto Biomecánico de Valencia (CSD/IBV, 2009). Los resultados mostraron que el porcentaje medio de accesibilidad fue del 50.6\%. Las zonas con mayor puntuación de accesibilidad fue el de salas y despachos (72\%), y ascensores ( $88 \%)$. En cambio, las zonas con menor accesibilidad fueron la recepción (13\%) y el comedor/restaurante/bar (33\%). Estos resultados revelan que la accesibilidad física no fue completa y que, por tanto, colectivos de personas con movilidad reducida podrían disminuir su participación en los programas deportivos ofertados al encontrar diferentes barreras de entrada en los espacios deportivos analizados. Se recomienda mejorar la accesibilidad física de los espacios deportivos ya que ayudaría a mejorar la integración y normalización de personas con discapacidad física en las actividades cotidianas de la sociedad.
\end{abstract}

Palabras clave: Gestión; pistas deportivas; evaluación; barreras; accesibilidad.

\begin{abstract}
The purpose of this study was to find out the mean percentage of physical accessibility of a group of conventional sports facilities in Extremadura, and also to determinate the percentage of accessibility of each different zone that constitutes these sports facilities. Specifically, 38 indoor courts from pavilions were analyzed (19 from the province of Caceres and 19 from the province of Badajoz), filling out 1673 items with the accessibility questionnaire proposed by the Spanish Sports Council and the Biomechanical Institute of Valencia (CSD/IBV, 2009). The results showed that the average percentage of accessibility was 50.6\%. The zones with the highest accessibility score were the rooms and offices (72\%), and elevators (88\%). On the contrary, the areas with the lowest accessibility rate were the reception (13\%), and the dining hall/restaurant/bar (33\%). These results revealed that physical accessibility is not complete, and therefore, the participation of groups of persons with reduced mobility in sport programmes offered by the local governments could decrease due to the existence of different accessibility barriers. It is recommended to improve the physical accessibility in sports facilities since it would increase the participation of people with reduced mobility in sports activities, and therefore, their integration and normalization in the daily activities of society.
\end{abstract}

Keywords: Management; sports facilities; evaluation; barriers; accessibility.

\section{Introducción}

La Organización Mundial de la Salud (OMS, 2001) establece que una persona con discapacidad es aquella que presenta algún problema en su estructura o función corporal, o bien dificultades para realizar acciones en el contexto social en el que vive, generando ciertas restricciones de participación en las tareas cotidianas. Para vencer esa visión negativa que conlleva en ocasiones el término discapacidad, el Sistema Internacional de Clasificación de Funcionalidad, Discapacidad y Salud (CIF) transformó una clasificación de la discapacidad basada en el déficit a una clasificación basada en las competencias de componentes de salud (i.e., cambiando el foco de atención al estado de salud y capacidades del usuario; ver Reina, 2014). Desde esta perspectiva, la discapacidad tendría que ver con todas aquellas actividades que la persona es capaz de hacer dentro de su contexto social de interacción. En cambio, personas con movilidad reducida serían aquellas personas que muestran una limitación, temporal o permanente, de sus posibilidades de desplazamiento o movimiento y precisan en consecuencia de una ayuda para la adquisición de bienes o servicios; e.g., un transporte público; ver ECMT, 2006). En España se consideran personas con discapacidad aquellas que presentan un grado de discapacidad superior o igual al 33\%, según el pro-

Fecha recepción: 26-03-20. Fecha de aceptación: 31-05-20 Vicente Luis del Campo viluca@unex.es cedimiento para reconocimiento, declaración y calificación del grado de discapacidad establecido por el Real Decreto 1971/1999, y posteriormente actualizado en el Real Decreto 1364/2012. La consideración de persona con discapacidad quedó establecida en la Ley 51/2003, modificada por el Real Decreto $1 / 2013$.

La última encuesta nacional publicada sobre «Discapacidad, Autonomía Personas y situaciones de Dependencia» por parte del Instituto Nacional de Estadística (INE, 2008), y a la espera de su actualización durante el año 2020, mostraba que España contaba con un total 3.85 millones de personas con algún tipo de discapacidad $(8.14 \%$ del total de población española). La edad media de las personas con discapacidad era de 64.3 años. Por género, 2.3 millones de mujeres afirmaban tener alguna discapacidad frente a 1.55 millones de hombres. Además, 1.2 millones de hogares españoles tenían personas con movilidad reducida y con barreras de acceso en sus edificios (e.g., escaleras sin rampas o sin plataformas móviles). En Extremadura, la cifra de personas con discapacidad alcanza el valor de 111000 personas (10.90\% total), siendo la segunda Comunidad Autónoma española con mayor tasa de dependencia. Posteriormente, el INE (2012) cuantificó en 4.3 millones las personas discapacitadas en España cuando la discapacidad estaba asociaba a la integración social de actividades de ocio. Según este organismo estatal, a través de una encuesta de la población activa y de la base Estatal de personas con discapacidad, el $36.6 \%$ de las personas con discapacidad legalmente reconocida eran activos. Además, 1.45 millones 
de personas entre los 16 y 64 años y residentes en hogares familiares tenían certificado de discapacidad. Esta cifra representa el $4.8 \%$ de la población española en edad laboral. En relación a la movilidad reducida, destacar que un $27 \%$ de las personas mayores de 74 años tenían limitaciones realizando actividades básicas mientras que el $81 \%$ de las personas con discapacidad señalaba barreras para salir de casa.

Más recientemente, el Observatorio Estatal para la Discapacidad muestra en el informe OLIVENZA (2017) que las personas con discapacidad tienen un $55 \%$ de desventaja en el acceso a derechos sociales tales como la participación política, educación, vivienda y salud en comparación con la población en general. Este porcentaje de desventaja alcanza el $73.3 \%$ cuando se refiere al acceso de actividades de ocio. En esta línea, el grado de participación e integración de las personas con discapacidad en diversos ámbitos de la sociedad (e.g. cultural, laboral, educativo, deportivo) requiere niveles adecuados de autonomía para la realización de sus desplazamientos cotidianos (Rodríguez de Luengo, 1999). Por tanto, el éxito en la consecución de una movilidad independiente, eficaz y segura está condicionado por el grado de accesibilidad del entorno. La accesibilidad ha sido descrita como una característica que permite el uso y disfrute de un entorno con independencia de su condición física, sensorial o intelectual (De Benito, García, Juncá, de Rojas \& Santos, 2005). Su ausencia impide que las personas con algún tipo de discapacidad puedan ejercer sus derechos individuales como persona, sustentando la creación de situaciones de dependencia (Asís \& Palacios, 2008).

En España existe numerosa normativa que regula la accesibilidad e integración de personas con discapacidad. Por ejemplo, la mencionada Ley 51/2003 del 2 diciembre, de igualdad de oportunidades, no discriminación y accesibilidad universal de las personas con discapacidad; o la Ley 39/ 2006, del 14 de diciembre, de promoción de la autonomía personal y atención a las personas en situación de dependencia. En Extremadura existe la Ley 11/2014, de 9 de diciembre, de accesibilidad universal de Extremadura que trata de regular una inclusión completa de las personas con discapacidad en diferentes ámbitos (e.g., acceso a los servicios públicos), reconociendo para ello fundamental la coordinación de acciones entre Administraciones públicas. Específicamente, esta Ley contempla la inclusión de medidas de acción positiva en el transporte, edificación, espacios públicos urbanizados y naturales para personas con discapacidad y movilidad reducida. Por ejemplo, la reserva de espacios y plazas de uso preferente para personas con discapacidad o movilidad reducida en locales de espectáculos, salas de conferencias o reuniones, etc. También, planes de autoprotección, emergencia y evacuación de instalaciones de uso o concurrencia pública.

A nivel deportivo, la accesibilidad también queda regulada a nivel nacional y autonómico. Por ejemplo, la Ley del Deporte (1990) afirma que todos los centros docentes, públicos o privados, deberán disponer de instalaciones deportivas accesibles y adaptadas a personas con movilidad reducida (Artículo 3, punto 3). Posteriormente, se especifica que las instalaciones deportivas de uso público deberán ser accesibles, y sin barreras ni obstáculos que imposibiliten la libre circulación de personas con minusvalía física o de edad avanzada (Artículo 70, punto 3). De igual forma, la Ley 2/ 1995, de 6 de abril, del Deporte de Extremadura (Capítulo III, artículo 70) establece que todas las instalaciones deportivas construidas en la Comunidad Autónoma de Extremadura deberán ser accesibles y sin barreras ni obstáculos que imposibiliten la libre circulación de personas con minusvalías o de edad avanzada.

Específicamente, la gestión y construcción de instalaciones deportivas para personas con necesidades especiales tiene como referencia básica la normativa europea UNE. Posteriormente, la Asociación Española de Normalización y Certificación (AENOR, 2006) desarrolla esta normativa en el estado español, a través de su Comité Técnico de Deportes, Equipamientos e Instalaciones Deportivas (AEN/CTN 147, SC5). Por ejemplo, la norma DALCO(UNE 170001-1: 2001) introdujo una serie de criterios relacionados con la accesibilidad (e.g., deambulación, aprehensión, localización y comunicación) a fin de posibilitar una movilidad independiente de las personas en todos los espacios de una instalación deportiva. También, el Consejo Superior de Deportes (CSD) ha venido coordinando desde el año 2009, en colaboración con el Instituto Biomecánico de Valencia (IBV), diferentes políticas de públicas de ordenación en la construcción de instalaciones deportivas desde el punto de vista de la accesibilidad.

Calder y Mulligan (2014) realizaron una revisión sistemática de instrumentos utilizados para medir la accesibilidad en centros deportivos fitness y de recreación. Estos autores encontraron siete instrumentos en diferentes bases de datos especializadas (e.g., MEDLINE, SCOPUS, SPORTDiscus, WOS), en un período temporal incluido entre 1950 y 2012. Los nombres de estos instrumentos fueron: $A D A$ Accessibility Guidelines Checklist for Buildings and Facilities (ADAAG); ADA Checklist for Readily Achievable Barrier Removal; Accessibility Instruments Measuring Fitness and Recreation Environments (AIMFREE); Community Health Environment Checklist-Mobility (CHEC-M); Removing Barriers to Health Clubs and Fitness Facilities (RBHCFF); Health Empowerment Zone Environmental Tool Shortened Environmental Checklist: Fitness Centre Survey (HEZEAT-FCS); Community Health Environment Checklist - Exercise Facilities (CHEC-Fit). Sin embargo, solamente el AIMFREE (Rimmer, Riley, Wang \& Rauworth, 2004) y el CHEC-M (Stark, Hollingsworth, Morgan $\&$ Gray, 2007) han sido testados para su desarrollo y validación.

En España, el análisis de la accesibilidad en instalaciones deportivas se hace a través de un test de accesibilidad diseñado por el CSD/IBV (2009). Se trata de un instrumento de evaluación que trata de conocer el grado de accesibilidad física que presentan diferentes espacios y elementos dentro de una instalación deportiva (e.g., exteriores, accesos, recepción, señalización e iluminación, circulaciones horizontales y pasillos, puertas interiores/escaleras, salas y despachos, aseos y vestuarios, comunicación, alarmas y evacuación, ascensores y piscina). Sin embargo, la valoración final de una instalación deportiva acerca de su grado de accesibilidad se ha reducido a conocer si esa instalación tenía o no barreras en acceso y circulaciones exteriores, barreras en circulaciones interiores, y vestuarios adaptados. De acuer- 
do con este planteamiento, una instalación deportiva sería accesible si: i) se puede acceder desde la calle (i.e., rampas con pendientes suaves y pasamanos), ii) se puede circular en el interior de los espacios (i.e., pasillos y puertas de anchura suficiente para el paso y giro de las sillas de ruedas) y iii) que haya vestuarios y servicios preparados para las personas con discapacidad.

La información acerca de la accesibilidad en los diferentes espacios deportivos es publicada por el CSD, cada cinco años, dentro del catálogo de publicaciones del censo nacional de instalaciones deportivas por Comunidad Autónoma, y en base a los datos aportados por los agentes censales de cada región. Las últimas publicaciones datan del año 2010, y están referidas a Castilla la Mancha, Extremadura y País Vasco. Para el resto de Comunidades, los últimos datos disponibles son del año 2005. Atendiendo a este análisis, Extremadura tendría 496 instalaciones totalmente accesibles y 411 no accesibles (ni para deportistas ni para espectadores). Estos valores representarían un $11.34 \%$ y un $9.39 \%$ del total de 4235 instalaciones censadas respectivamente (Monzú, 2013).

Actualmente, apenas existen estudios en España que hayan analizado el grado de accesibilidad física de las instalaciones deportivas a partir del test de accesibilidad del CSD/ IBV (2009). Como excepción, Gallardo, Felipe, Burillo, García, Plaza y Sánchez (2009) analizaron el porcentaje de accesibilidad en espacios deportivos de 20 centros escolares de la Comunidad Autónoma de Castilla la Mancha. Los resultados de dicho estudio reflejaron un porcentaje de accesibilidad similar entre espacios deportivos cerrados $(60 \%$ en pabellones polideportivos) y abiertos $(57.14 \%$ en pistas polideportivas). Por tanto, existe un limitado y no actualizado conocimiento acerca del grado de accesibilidad física que presentan los espacios deportivos en el territorio nacional.

En el presente estudio se decide analizar las pistas y pabellones polideportivos de Extremadura ya que fueron el grupo de espacios deportivos convencionales más numeroso en esta Comunidad Autónoma, con un total de 1602 espacios deportivos censados (28.49\%); de las cuales 1330 pistas fueron en espacio abierto (23.65\%), y 266 pistas fueron en espacio cerrado (4.73\%). Específicamente, se analizan los espacios cerrados ya que casi dos terceras partes del total de Municipios de Extremadura cuentan con pistas y/o pabellones en recinto cerrado (Monzú, 2013). Partiendo de estas premisas, los objetivos del estudio fueron: i) conocer el porcentaje de accesibilidad física que presentaría un conjunto de pistas polideportivas cubiertas en Extremadura, de acuerdo al test de accesibilidad (CSD/IBV, 2009), e ii) identificar qué espacios de estas instalaciones presentan más barreras u obstáculos para las personas con discapacidad física.

\section{Método}

\section{Muestra}

Para la realización de este estudio se analizaron 38 pistas deportivas interiores de pabellones en la Comunidad Autónoma de Extremadura, de las cuales 19 fueron de Municipios de la provincia de Badajoz y las otras 19 de Municipios de la provincia de Cáceres. La identidad de estos espacios deportivos, así como su pertenencia a un Municipio extremeño se mantuvo en el anonimato a fin de preservar la confidencialidad de la información analizada. Este dato representó el $14.28 \%$ del total de espacios deportivos en recinto cerrado de pistas y pabellones polideportivos en Extremadura(Monzú, 2013).

\section{Material}

El instrumento de recogida de datos utilizado para evaluar la accesibilidad de las instalaciones fue el test de control para la accesibilidad de una instalación deportiva del CSD/ IBV (2009). Este test de control aparece por primera vez en el marco del proyecto nacional para la gestión de riesgos en las instalaciones deportivas impulsado por el propio CSD (2009), y como consecuencia de las necesidades anteriores detectadas en el proyecto nacional de mejora y armonización de las instalaciones deportivas en España (proyecto MAID). La finalidad del test de control para la accesibilidad, junto con el de seguridad, fue generar conocimiento acerca de la situación actual de las instalaciones deportivas para así dotar de un procedimiento posterior de mejora en materia de accesibilidad y seguridad (e.g., listado de actuaciones preventivas o correctivas a realizar). El test de control para la accesibilidad está basado en documentos técnicos (normas) de referencia provenientes del código técnico de la edificación, reglamentos de algunas federaciones deportivas, normas UNE-EN, y de la legislación autonómica en materia de accesibilidad (CSD/ IBV, 2010). Por tanto, este test de control se desarrolla a partir de las diferentes indicaciones que las normas especifican, descomponiéndolas en ítems sencillos de aplicar.

El test de control para la accesibilidad se trata de un cuestionario a modo de check list que se divide en 13 bloques de ítems. Cada ítem contiene una oración con un enunciado afirmativo respecto a un aspecto particular de la accesibilidad física de la instalación deportiva (e.g., ítem 1. Dispone de parada de transporte púbico). Los bloques de ítems fueron: 1. Exterior (4 ítems), 2. Accesos (3 ítems), 3. Recepción (3 ítems), 4. Señalización e iluminación (3 ítems), 5. Circulaciones horizontales y pasillos (4 ítems), 6. Puertas interiores y escaleras (5 ítems), 7. Salas y despachos (4 ítems), 8. Cuartos de baño y vestuarios (10 ítems), 9. Comunicación, alarmas y evacuación (3 ítems), 10. Comedor/restaurante/bar (5 ítems), 11. Ascensores (4 ítems), 12. Piscina (2 ítems), y 13. Graderíos e instalaciones para espectadores (4 ítems). Este check list ha sido previamente utilizado por Gallardo et al. (2009) para analizar la accesibilidad de espacios deportivos docentes. De acuerdo a estos autores, la utilización de un check list permitiría obtener una visión general del estado de la instalación, seguimiento y detección de necesidades de mejora, adecuación a la normativa vigente y, el uso de un formato sencillo de análisis de las variables de estudio.

El procedimiento de medida se basó en una recogida in situ de datos. La recogida de datos fue realizada los meses de marzo, abril y mayo de 2018 debido a la disponibilidad temporal del equipo investigador. Inicialmente se realizó una inspección visual de la instalación deportiva, centrada en realizar una exploración inicial de dicho espacio deportivo. A continuación, se procedió a evaluar específicamente la pista deportiva cerrada en base al test de control de accesibilidad.

\section{Variables}

La variable dependiente del estudio fue el porcentaje 
medio de accesibilidad del conjunto de pistas polideportivas cerradas. Si la instalación deportiva analizada cumplía con el enunciado de accesibilidad expuesto en el ítem, entonces se respondía con «Sí». En cambio, si no cumplía con el enunciado se respondía con «No». Destacar que cada ítem incluía las dos opciones de respuesta («Sí» o «No»), así como una breve descripción de cómo debería ser la instalación, a nivel de accesibilidad física, en ese aspecto concreto. Por ejemplo, el primer enunciado del bloque de Recepción (ítem $n^{\circ} 8$ ) exponía una cuestión acerca del mostrador de recepción. Si el mostrador de la pista cerrada cumplía con las recomendaciones de accesibilidad marcadas en la descripción (i.e., tener una zona de atención de altura de $.80 \mathrm{~cm}$ y una altura inferior libre de $.70 \mathrm{~cm}$ ), entonces ese ítem era respondido con «Sí» (y codificado para el análisis con 1). Por el contrario, si ese ítem no cumplía con la recomendación expuesta se respondía con «No» (y codificado para el análisis con 0 ).

Para calcular la variable dependiente se consideró la relación de ítems tipificados con 1 respecto al total de ítems respondidos con 0 o 1 . Para facilitar su comprensión, el porcentaje de accesibilidad fue expresado posteriormente respecto al $100 \%$. Por ejemplo, si un espacio deportivo alcanzó el 50\% de accesibilidad significaba que la mitad de los ítems fueron respondidos con un 1 y la otra mitad con 0 . Para un análisis más preciso del grado de accesibilidad de estas pistas deportivas cerradas se consideró como variable independiente los diferentes grupos de ítems que componen el check list del cuestionario (CSD/IBV, 2009). Por tanto, como dicho test de control contaba con 13 grupos de ítems, la variable independiente del estudio tuvo 13 niveles a fin de conocer el porcentaje de accesibilidad en cada bloque de ítems y su comparación respecto al resto de bloques.

\section{Diseño}

El planteamiento del problema se abordó desde la perspectiva de la investigación social, a través de la aplicación de una metodología descriptiva de carácter cuantitativa y transversal. La recogida de los datos se realizó: i) en un entorno real y natural donde no existió manipulación del entorno, y ii) por observación estructurada, utilizando como técnica de investigación el cuestionario (Thomas \& Nelson, 2007). Además, se trató de un diseño cuasi-experimental al no existir una manipulación real de la variable independiente (Pereda, 1987). La muestra de instalaciones deportivas fue seleccionada por provincia a través del muestreo aleatorio simple en el que el equipo investigador asignó un número a cada Municipio, y a través de un programa generador de números aleatorios, se seleccionaron los Municipios participantes. El número total de pistas deportivas analizadas fue determinado atendiendo a la disponibilidad del equipo investigador.

\section{Análisis de datos}

En primer lugar, se realizó la prueba de KolmogorovSmirnov para conocer si la variable de estudio presentaba una distribución normal de valores. Como el análisis de la accesibilidad se trató en cada ítem del cuestionario de forma dicotómica $(0=$ no presenta accesibilidad, $1=$ si presenta accesibilidad), la prueba estadística confirmó una falta de distribución normal de los datos en la variable dependiente.
Se solicitaron estadísticos descriptivos, según bloque de ítems, a fin de conocer el porcentaje medio de accesibilidad y el rango promedio de accesibilidad.

La muestra inicial de datos a recoger ascendía a 2052 valores (38 pistas analizadas x 54 ítems del cuestionario de accesibilidad del CSD/IBV, 2009). Sin embargo, 1673 ítems fueron finalmente cumplimentados ya que no todas las pistas seleccionadas tenían todos los espacios o elementos para poder ser respondidos (e.g., muchas carecían de ascensores o piscina y, por tanto, dichos ítems no pudieron ser respondidos, perdiendo esos datos para el análisis).

Se realizó la prueba no paramétrica de Kruskal-Wallis para comparar los rangos promedio de accesibilidad entre bloques de ítems. Confirmadas las diferencias, se realizó la prueba no paramétrica de U de Mann-Whitney para ver en qué pares de bloques de ítems existían diferencias de rangos. Específicamente, esta comparación de pares se realizó entre los siete primeros bloques de ítems del cuestionario ya que estos bloques de ítems reunían el número de casos válidos suficientes para realizar la prueba estadística. Se solicitó un nivel Alpha de $p<.05$ para este análisis. El análisis estadístico fue realizado con el paquete estadístico 18.0 SPSS (C) 2008 SPSS Inc.).

\section{Resultados}

En primer lugar, destacar que los bloques de ítems que alcanzaron un mayor porcentaje de accesibilidad (>70\%) y un rango promedio superior a 1000 fueron los ascensores, salas y despachos. En cambio, los espacios menos accesibles del conjunto de pistas deportivas interiores analizadas, con un porcentaje inferior al $40 \%$ y un rango promedio menor a 700, fueron las zonas de recepción, gradas, y restauración (ver tabla 1). La media total de accesibilidad, independientemente del bloque de ítems, fue del 50.6\%.

\begin{tabular}{|c|c|c|}
\hline Bloque de ítems & $\mathrm{M}(\mathrm{DT})$ & Ranoo nromedio \\
\hline Exterior & $56 \%(49.8)$ & 881.78 \\
\hline Accesos & $57 \%(49.7)$ & 890.95 \\
\hline Recepción & $13 \%(34.1)$ & 525.04 \\
\hline Señalización e iluminación & $63 \%(48.6)$ & 936.81 \\
\hline Circulaciones horizontales y pasillos & $43 \%(49.6)$ & 771.71 \\
\hline Puertas interiores y escaleras & $61 \%(49)$ & 920.30 \\
\hline Salas y despachos & $72 \%(44.9)$ & 1019.36 \\
\hline Cuartos de baño y vestuarios & $56 \%(49.7)$ & 884.12 \\
\hline Comunicación, alarmas y evacuación & $37 \%(48.5)$ & 724.91 \\
\hline Comedor/restaurante/bar & $33 \%(47.9)$ & 692.83 \\
\hline Ascensores & $88 \%(35.4)$ & 1145.94 \\
\hline Piscina & $68 \%(47.7)$ & 984.34 \\
\hline Graderíos e instalaciones para espectadores & $35 \%(43.5)$ & 623.13 \\
\hline
\end{tabular}

La prueba Kruskal-Wallis determinó que existían diferencias entre bloques de ítems $\left(x^{2}(1,12)=17.208 ; p<.001\right)$. Específicamente, el bloque de ítems relativas a los exteriores $\mathrm{y}$ accesos de la instalación tuvieron significativamente un mayor rango promedio que la recepción; circulaciones y pasillos; salas y despachos; y comunicación, alarmas y evacuación.

Destacar también que la zona de recepción obtuvo un menor rango promedio que la zona de señalización e iluminación; circulaciones y pasillos; puertas interiores y escaleras; salas y despachos; cuartos de baños y vestuarios; y comunicaciones, alarmas y evacuación. Similarmente, la zona de circulaciones y pasillos tuvo un menor rango que puertas interiores y escaleras; salas y despachos; cuartos de baños 
Estadístico U Mann de Whitney para aquellas comparaciones de pares de bloques de ítems con diferencias significativas en el rango promedio de accesibilidad en el conjunto de pistas deportivas cubiertas analizadas en Extremadura

\begin{tabular}{|c|c|c|c|c|c|c|c|c|c|c|c|c|c|}
\hline Bloq. Preg. & (1) & (2) & (3) & (4) & (5) & (6) & (7) & (8) & (9) & (10) & (11) & (12) & (13) \\
\hline (1) & - & - & $4925.5^{* * *}$ & - & $10032^{*}$ & - & $9652 * *$ & - & $6977.5^{* *}$ & $1765^{*}$ & - & - & $1305^{*}$ \\
\hline (2) & - & - & $3623.5^{* * *}$ & - & $7429 *$ & - & $7334 * *$ & - & $5162.5^{* *}$ & $7140^{* * *}$ & - & - & $5270^{* * *}$ \\
\hline (3) & $4925.5^{* * *}$ & $3623.5^{* * *}$ & - & $3213^{* * *}$ & $6055^{* * *}$ & $5662.5^{* * *}$ & $3513 * * *$ & $12221.5^{* * *}$ & $4859^{* * *}$ & $1355^{*}$ & $116.5^{*}$ & - & 6783* \\
\hline (4) & - & - & $3213 * * *$ & - & $6832 * *$ & - & - & - & $4725^{* * *}$ & $1190^{* *}$ & - & - & $4760^{* * * *}$ \\
\hline (5) & $10032 *$ & $7429 *$ & $6055 * * *$ & $6832^{* *}$ & - & $11875^{* *}$ & $18175.5^{* * *}$ & $56932.5^{* * *}$ & - & - & $510^{*}$ & $3660^{* *}$ & - \\
\hline (6) & - & - & $5662.5^{* * *}$ & - & $11875^{* *}$ & - & - & - & - & $8920^{* * *}$ & - & - & $18614^{* * *}$ \\
\hline (7) & $9652^{* *}$ & $7334 * *$ & $3513 * * *$ & - & $18175.5^{* * *}$ & - & - & $55269.5^{* * *}$ & $12518^{* * *}$ & $5835^{* * *}$ & - & - & $11719.5^{*}$ \\
\hline (8) & - & - & $12221.5^{* * *}$ & - & $56932.5^{* * *}$ & - & $55269.5^{* * *}$ & - & - & - & - & - & - \\
\hline (9) & $6977.5^{* *}$ & $5162.5^{* *}$ & $4859^{* * *}$ & $4725^{* * *}$ & - & - & $12518^{* * *}$ & - & - & - & - & - & - \\
\hline (10) & $1765^{*}$ & $7140 * * *$ & $1355^{*}$ & $1190^{* *}$ & - & $8920^{* * *}$ & $5835^{* * *}$ & - & - & - & - & - & - \\
\hline (11) & - & - & $116.5^{*}$ & - & $510^{*}$ & - & - & - & - & - & - & - & - \\
\hline (12) & - & - & - & - & $3660^{* *}$ & - & - & - & - & - & - & - & - \\
\hline (13) & $1305^{*}$ & $5270 * * *$ & $6783^{*}$ & $4760^{* * *}$ & - & $18614 * * *$ & $11719.5^{*}$ & - & - & - & - & - & - \\
\hline
\end{tabular}

y vestuarios; y piscina. En esta línea, tanto la sala de comedor/restaurante/bar como las gradas tuvieron menos accesibilidad que el exterior; accesos; recepción; señalización e iluminación; puertas interiores y escaleras; y salas y despachos (ver tabla 2).

En cambio, las zonas de señalización e iluminación tuvieron más accesibilidad que las circulaciones y pasillos; y comunicaciones, alarmas y evacuación. También, el ascensor alcanzó mayor rango que las zonas de circulaciones y pasillos; y recepción. Por último, las salas y despachos tuvieron más rango promedio que cuartos de baño y vestuarios; y comunicaciones, alarmas y evacuación.

\section{Discusión}

El objetivo del estudio fue conocer el nivel de accesibilidad física que presentan una muestra reducida de pistas deportivas cerradas en Extremadura, utilizando el test de control de accesibilidad del CSD/IBV (2009). Destacar el valor del presente estudio ya que, más allá de que sus datos estén referenciados a una región española y a un tipo concreto de espacio deportivo convencional, no existían evidencias previas acerca del porcentaje de accesibilidad física en instalaciones deportivas municipales en España. El único antecedente similar se encuentra en el estudio de Gallardo et al. (2009) cuando analizaron la accesibilidad física de diferentes instalaciones deportivas docentes en la Comunidad Autónoma de Castilla la Mancha.

Destacar que la accesibilidad física alcanzó una media final ligeramente superior al 50\%, lo que significa que casi la mitad de los enunciados expuestos en el check list fueron respondidos negativamente en términos de accesibilidad (i.e., las pistas interiores no fueron accesibles en casi un 50\% de los ítems respondidos). El porcentaje de ítems con menor porcentaje de accesibilidad se encuentra en la recepción que es aquella zona de la instalación donde se recibe y atiende a los clientes. Destacar también que la segunda zona menos accesible fue el graderío. Con estos datos, sugerimos que el diseño de las pistas analizadas fue concebido más para la práctica deportiva que para la observación de la actividad deportiva. En cambio, las zonas de la instalación más accesibles fueron los ascensores y las salas y despachos.

Independientemente de los porcentajes de accesibilidad conseguidos en cada bloque de ítems, estos resultados coinciden con estudios anteriores al señalar que las instalaciones deportivas no tienen una accesibilidad completa. Por ejemplo, Rimmer, Riley, Wang y Rauworth (2005) concluyeron que 35 centros de salud y de fítness americanos tuvieron niveles bajos y moderados de accesibilidad. Riley, Rimmer,
Wang y Schiller (2008) mostraron que los accesos a las instalaciones deportivas fueron las áreas con mayor accesibilidad (70 sobre 100 puntos) mientras que la recepción sólo alcanzó una media de 39.61 puntos. Recientemente, Rimmer, Padalabalanarayanan, Malone y Mehta (2017) estudiaron 227 instalaciones de fítness de 10 Estados Americanos, obteniendo valores menores a 70 sobre 100 puntos en 11 de las 13 secciones del instrumento. En Canadá, ArbourNicitopoulos y Martin-Ginis (2011) cuantificaron una accesibilidad limitada para personas con problemas de movilidad (e.g., valores comprendidos entre 31 y 63 sobre 100 puntos) en 44 instalaciones deportivas de fitness (interiores) y recreación (exteriores). Destacar que las instalaciones de recreación tuvieron mayores niveles de accesibilidad que las de fitness.

Estos estudios evidencian que un nivel bajo de accesibilidad en instalaciones deportivas ya sea en espacios deportivos convencionales o de otro tipo (e.g., parques al aire libre o de juego), podría limitar las oportunidades de participación en personas con limitaciones físicas o de movilidad (Rimmer \& Rowland, 2008), y aumentar su riesgo de adquirir hábitos de vida sedentarios (Altman \& Bernstein, 2008). Entre las causas que pueden limitar el grado de participación de personas con discapacidad en actividades físicas se encuentra un conjunto multifactorial de barreras. Por ejemplo, el entorno natural o artificial donde se construyen las instalaciones; aspectos políticos y económicos; factores emocionales y psicológicos; equipamiento utilizado; interpretación de la normativa; falta de información, conocimiento y formación en torno a la discapacidad; $y$ las percepciones y actitudes de las personas no discapacitadas (Rimmer, Riley, Wang, Rauworth \& Jurkowski, 2004).

Quisiéramos destacar este último factor como una barrera relevante de acceso de personas con discapacidad a las instalaciones y espacios deportivos. Según Rowland, Fragala-Pinkham, Miles y O’Neil (2015), entre las causas que pudieron limitar la participación de personas con discapacidad en programas de actividad física se encuentran el propio comportamiento de los trabajadores de las instalaciones deportivas, así como su falta de conocimiento o deseo por desarrollar adaptaciones hacia estos colectivos. En esta línea, Alcaraz, Caballero, Sáenz-López y Fernández (2019) identificaron la falta de información, formación y conocimiento de las federaciones, organización y técnicos como barreras percibidas por deportistas con diversidad funcional visual y sus guías durante carreras de montaña celebradas en espacios deportivos no convencionales, abiertos y realizados en entornos naturales.

En el contexto educativo, González y Macías (2018) des- 
tacaron la importancia de la formación permanente del profesor de educación física para impulsar la inclusión social del alumnado con discapacidad física. Por ejemplo, realizando proyectos de innovación e investigación en educación física y atención a la diversidad, creación de recursos TIC y materiales adaptados para el trabajo con alumnos con discapacidad física. Similarmente, Canales, Aravena, Carcamo-Oyarzun, Lorca y Martínez-Salazar (2018) encontraron que las habilidades personales del profesorado fue una de las prácticas pedagógicas que favorecen la inclusión educativa durante las clases de educación física. Y es que la actividad físico-deportiva podría convertirse en un recurso práctico, sencillo y eficaz, para conseguir una acción docente más inclusiva y transformadora (Felipe-Rello, Garoz \& Tejero-González, 2020). Por ejemplo, Muñoz, Smith y Matos (2020) observaron una mejora de las relaciones sociales y participación entre estudiantes universitarios del Grado en Ciencias de la Actividad Física del Deporte y personas con discapacidad después de participar conjuntamente de una experiencia de inclusión educativa.

La accesibilidad se evidencia en acciones, pero también en actitudes que compensen la discapacidad, permitiendo desarrollar la capacidad de la persona mediante un diseño de todo para todos (Luque, Rodríguez \& Romero, 2005). En este diseño universal, la accesibilidad no sólo se refiere a facilitar el acceso físico (i.e., eliminar barreras arquitectónicas) sino también a las posibilidades de comunicarse con el medio circundante, a las condiciones que facilitan la instalación, uso de la tecnología y del transporte. Por tanto, para evaluar la accesibilidad de espacios y/o instalaciones se ha de tener en cuenta la calidad del diseño, la funcionalidad, la seguridad, la comodidad y la innovación, pero también la vida independiente y la normalización (Guillamón, 2003).

En nuestra opinión es muy importante que los responsables políticos, gestores y trabajadores de centros deportivos, tanto públicos como privados, tomen conciencia de la necesidad de normalizar las instalaciones deportivas desde el punto de la accesibilidad. Esta normalización de los espacios deportivos permitiría no sólo un acceso mayor de personas con algún tipo de discapacidad física a las instalaciones sino también un mayor uso por parte de personas con algún tipo de movilidad reducida (e.g., niños y personas mayores). Por ejemplo, Montilla (2019) realizó una intervención con un grupo reducido de gimnastas con discapacidad intelectual. Esta experiencia incluyó modificaciones en el reglamento, estrategias de intervención, y organización de ejercicios a fin de disminuir las barreras de participación en los entrenamientos y exhibiciones del club; y proveer junto a la Federación Catalana de Gimnasia de un reglamento específico de competición para gimnastas con discapacidad intelectual. En esta línea, una mejora en la accesibilidad física repercutiría en una mayor sostenibilidad social de esos espacios deportivos al haber más colectivos y personas practicando actividad física en su interior.

Defendemos que las medidas de accesibilidad en instalaciones deportivas sean consideradas siempre como una buena práctica, tal como establece el CSD/IBV (2009), evitando que cualquier tipo de barrera suponga un impedimento para la correcta utilización de esa instalación (Blumenau \& Rovira, 1996; Martínez-Tur, Tordera \& Ramos, 1996; Moreno-Luzón,
2001). Sin duda, la existencia de una normativa aplicable de accesibilidad en las instalaciones deportivas podría ayudar a reducir los obstáculos de entrada para las personas con discapacidad física. Por ejemplo, en Estados Unidos, aquellas instalaciones deportivas que fueron construidas después de la aprobación de la Ley Estadounidense de discapacidad (ADA) tuvieron mayores niveles de accesibilidad que aquellas que fueron construidas previamente a esta normativa (Rimmer et al., 2017).

Al tratarse de un estudio exploratorio, la limitación principal del estudio se encuentra en el reducido número de pistas deportivas analizadas en comparación con el total existente en Extremadura. Próximos estudios deberían aumentar el número de estos espacios deportivos para conseguir resultados representativos en materia de accesibilidad física en instalaciones deportivas. De acuerdo con las fórmulas de distribución gaussiana (y su asunción de distribución normalizada de los datos), la estimación del tamaño de una muestra representativa para la población total de pistas polideportivas cerradas en Extremadura debería ser 54 pistas (con un nivel de confianza del 95\% y un error del 5\%).

En el futuro, con una muestra mayor de instalaciones deportivas, sería interesante hacer análisis más concretos de variables contextuales que podrían condicionar el grado de accesibilidad en los espacios deportivos. Por ejemplo, ¿influye el tamaño de la población en la accesibilidad de las instalaciones deportivas?; es decir, ¿son más accesibles las instalaciones deportivas de las ciudades más grandes de Extremadura en comparación con aquellas instalaciones ubicadas en pequeños Municipios o viceversa? Similarmente, ¿influye el tipo de gestión (pública vs privada) en la accesibilidad de las instalaciones deportivas? En esta línea, para conseguir un análisis más completo de la accesibilidad física como elemento de calidad en las instalaciones deportivas se debería incluir también una metodología no sólo cuantitativa sino también cualitativa a fin de identificar las necesidades que personas con discapacidad perciben en el acceso y uso de los diferentes tipos de espacios deportivos. Esta información podría tener una gran utilidad en las decisiones que el grupo interdisciplinar de profesionales (arquitectos, gestores y técnicos deportivos, políticos del área de deportes) podría tomar al respecto del diseño de las instalaciones deportivas desde el punto de vista de la accesibilidad física.

Por último, se podría analizar no sólo la accesibilidad física sino también la accesibilidad desde el punto de vista sensorial ya que la mayoría de los estudios realizados se han enfocado a personas con movilidad reducida. En esta línea, López y Alejandre (2006) apuntan que existe una deficiente señalización en los accesos a las instalaciones deportivas, con ausencia de carteles accesibles y de contrastes de color entre puertas y paredes. Por ejemplo, las medidas de accesibilidad en instalaciones deportivas para personas con diversidad funcional visual suelen ser deficientes o incorrectas ya que se trata de un colectivo heterogéneo que requeriría diferentes y específicas adaptaciones (De Potter, 2006).

\section{Conclusiones}

Los resultados encontrados en este estudio hay que tomarlos con precaución ya que no se trata de una muestra 
representativa del conjunto de pistas cerradas de Extremadura sino solamente de un número reducido de dichos espacios deportivos convencionales. Esta circunstancia limita la capacidad de generalizar los resultados. Destacar, en todo caso, que la muestra analizada de pistas polideportivas cubiertas en Extremadura no fue $100 \%$ accesible, desde el punto de vista físico, ya que alcanzó un 50.6\% de accesibilidad según el cuestionario de accesibilidad del CSD/IBV (2009). Los espacios con mayor porcentaje de accesibilidad en estas instalaciones deportivas convencionales fueron los ascensores (88\%), salas y despachos (72\%). En cambio, los espacios menos accesibles fueron la recepción (13\%) y el graderío (35\%).

\section{Aplicaciones prácticas}

Tal como sugieren muchos estudios, la falta de accesibilidad física en las instalaciones deportivas podría constituir una barrera de entrada para la participación deportiva de las personas con discapacidad y movilidad reducida, limitando sus oportunidades de integración en la sociedad. En esta línea, el estudio realizado constituye una oportunidad para tomar consciencia de esta situación que afecta a millones de personas en España. Apostamos por el desarrollo de informes técnicos que analicen la accesibilidad física de los espacios deportivos, y que sean realizados por los servicios técnicos deportivos de las diferentes Administraciones Públicas o bien por empresas especializadas en la gestión de instalaciones deportivas. En nuestra opinión, dichos informes deberían estar encaminados a identificar qué zonas de los espacios deportivos presentan un menor grado de accesibilidad para así posteriormente implementar las medidas correctoras necesarias, de acuerdo a un sistema de prioridades previamente establecido (e.g., ¿es más importante que haya una rampa de acceso con una ligera pendiente en la puerta de acceso o encontrar un tirador grande en la puerta de entrada al lavabo?). En esta línea, el propio de test de control de accesibilidad del CSD/IBV (2009) atribuye a cada ítem una prioridad alta o media. Se podría seguir esta referencia inicial para evaluar qué aspectos de la instalación deportiva, en caso de no ser accesibles, deberían ser antes o después adaptados a fin de cumplir con la normativa específica. Además, en las instalaciones deportivas de nueva creación, la existencia de una cultura de análisis de la accesibilidad fomentaría el uso de diseños funcionales para toda la población por parte del equipo interdisciplinar encargado de proyectar la instalación (i.e., arquitecto, empresa de construcción, gestor deportivo). Pensar un diseño de instalación deportiva para ser disfrutada por todos sería de gran interés para aquellos grupos de personas con algún tipo de discapacidad física o movilidad reducida (e.g., ancianos, niños) a fin de garantizarles una deambulación independiente por todas las zonas de la instalación (e.g., deportivas, baños y aseos, pasillos, entrada, grada).

\section{Referencias}

AENOR. (2006). Normas europeas en materia de deportes. Madrid: Consejo Superior de Deportes y AENOR. Alcaraz, V., Caballero, P., Sáenz-López, P., \& Fernández, J.
(2019). Barreras percibidas por deportistas con diversidad funcional visual y guías en carreras por montaña. Retos, 36, 107-112.

Altman, B. M., \& Bernstein, A. B. (2008). Disability and Health in the United States, 2001-2005. Hyattsville, MD: National Center for Health Statistics.

Arbour-Nicitopoulos, K. P., \& Martin-Ginis, K. A. (2011). Universal accessibility of accessible fitness and recreational facilities for person whit mobility disabilities. Adapted Physical Activity Quarterly, 28, 1-15.

Asís, R., \& Palacios, A. (2008). Derechos humanos y situaciones de dependencia. España: Dykinson.

Blumenau, K., \& Rovira, E. (1996). Instalaciones deportivas sin barreras. Málaga: Junta de Andalucía. Instituto Andaluz del Deporte.

Calder, A. M., \& Mulligan, H. F. (2014). Measurement properties of instruments that assess inclusive access to fitness and recreational sports centers: A systematic review. Disability and Health Journal, 7(1), 26-35. doi: 10.1016/j.dhjo.2013.06.003

Canales, P., Aravena, O., Carcamo-Oyarzun, J., Lorca, J., \& Martínez-Salazar, C. (2018). Prácticas pedagógicas que favorecen u obstaculizan la inclusión educativa en el aula de educación física desde la perspectiva del alumnado y profesorado. Retos, 34, 212-217.

CSD/IBV. (2009). Buenas prácticas en Instalaciones Deportivas. Madrid: Consejo Superior de Deportes (CSD), Instituto de Biomecánica de Valencia (IBV), y Federación Española de Municipios y Provincias (FEMP).

CSD/IBV. (2010). Legislación y documentos técnicos de referencia en instalaciones deportivas. Madrid: Consejo Superior de Deportes (CSD), e Instituto de Biomecánica de Valencia (IBV).

De Benito, J., García, J., Juncá, J. A., de Rojas, C., \& Santos, J. J. (2005). Manual para un entorno accesible. Madrid: Real Patronato sobre Discapacidad.

De Potter J. C. (2006). The contribution of sport and physical activity to the-wellbeing of visually impaired people. Science \& Sport, 21, 249-250.

ECMT. (2006). Improving Transport Accessibility for All. European Conference of Ministers of Transport. OECD Publications Service. París. France.

Felipe-Rello, C., Garoz, I., \& Tejero-González, C. M. (2020). Cambiando las actitudes hacia la discapacidad: diseño de un programa de sensibilización en Educación Física. Retos, 37, 713-721.

Gallardo, L., Felipe, J. L., Burillo, P., García, M., Plaza, M., \& Sánchez, J. (2009). Análisis de la seguridad y accesibilidad en instalaciones deportivas de centros escolares. Fundación Mapfre.

González, I., \& Macías, D. (2018). La formación permanente como herramienta para mejorar la intervención del maestro de educación física con alumnado con discapacidad. Retos, 33, 118-122.

Guillamón, J. (2003). La accesibilidad, reto profesional y exigencia social. Ingeniería y Territorio, 63, 4-9.

INE (2008). Encuesta de Discapacidad, Autonomía personal y situaciones de Dependencia (EDAD) 2008. Recuperado de http://www.ine.es/prensa/np524.pdf

INE (2012). El empleo de las personas con discapacidad 
2012. Recuperado de http:// www.ine.es/metodologia/ t22/informe_2012.pdf

Ley 10/1990, de 15 de octubre, del Deporte. Boletín Oficial del Estado, núm. 249, pp. 30397-30411.

Ley 2/1995, de 30 de mayo, del Deporte de Extremadura. Boletín Oficial del Estado, núm. 128, pp. 15700-15713.

Ley 51/2003, de 2 de diciembre, de igualdad de oportunidades, no discriminación y accesibilidad universal de las personas con discapacidad. Boletín Oficial del Estado, núm. 289, pp. 43187-43195.

Ley 39/2006, de 14 de diciembre, de Promoción de la Autonomía Personal y Atención a las personas en situación de dependencia. Boletín Oficial del Estado, núm. 299, pp. $44142-44156$.

Ley 11/2014, de 9 de diciembre, de accesibilidad universal de Extremadura. Diario Oficial de Extremadura, núm. 239, pp. 37307-37335.

López, A., \& Alejandre, A. (2006). La accesibilidad arquitectónica para personas con discapacidades sensoriales en los espacios deportivos de Asturias. Universidad de León. Revista Digital EF deportes- Buenos Aires, 103. Recuperado de: http://www.efdeportes.com/efd103/ accesib.htm

Luque, D. J., Rodríguez, G., \& Romero, J.F. (2005). Accesibilidad y Universidad. Un estudio descriptivo. Intervención Psicosocial, 14(2), 209-222.

Martínez-Tur, V., Tordera, N., \& Ramos, J. (1996). Tipología de instalaciones deportivas en función de su carácter público o privado: diferencias en la gestión y uso de las mismas. Apunts: Educación Física y Deportes, 43, 91102.

Montilla, M. J. (2019). Inclusión y competición: Adaptaciones para disminuir las barreras de participación de un grupo de gimnastas con discapacidad intelectual en gimnasia rítmica y propuesta de reglamento específico para la competición. Retos, 36, 552-560.

Monzú, J. P. (2013). Censo Nacional de Instalaciones Deportivas del 2010: Extremadura. Madrid: Consejo Superior Deportes y Junta de Extremadura.

Moreno-Luzón, M. (2001). Gestión de la calidad y diseño de organizaciones. Barcelona: Gestión 2000.

Muñoz,A., Smith, E., \& Matos, M. (2020). Una experiencia de inclusión educativa en el aula de Expresión Corporal con alumnado universitario. Retos, 37, 702-705.

Observatorio Estatal para la Discapacidad (2017). Informe Olivenza 2017, sobre la situación general de la discapacidad en España. Antonio Jiménez Lara y Agustín Huete García (Coords.). Recuperado de: https:/ /www.observatoriodeladiscapacidad.info/informeolivenza-2017-2/

OMS (2001). Clasificación Internacional del Funcionamiento, de la Discapacidad y de la Salud. Madrid: Ministerio de Trabajo y Asuntos Sociales.

Pereda, S. (1987). Psicología Experimental. I Metodología. Madrid: Ediciones Pirámide.

Real Decreto 1971/1999, de 23 de diciembre, de procedimiento para el reconocimiento, declaración y calificación del grado de minusvalía. Boletín Oficial del Estado, núm. 22,pp. 3317-3410.

Real Decreto 1364/2012, de 27 de septiembre, por el que se modifica el Real Decreto 1971/1999, de 23 de diciembre, de procedimiento para el reconocimiento, declaración y calificación del grado de discapacidad. Boletín Oficial del Estado, núm. 245, pp. 72772-72774.

Real Decreto 1/2013, de 29 de noviembre, por el que se aprueba el Texto Refundido de la Ley General de derechos de las personas con discapacidad y de su inclusión social. Boletín Oficial del Estado, núm. 289, pp. 95635-95673.

Reina, R. (2014). Inclusión en deporte adaptado: dos caras de una misma moneda. Psychology, Society and Education, 6(1), 55-67.

Riley, B., Rimmer, J., Wang, E., \& Schiller, W. (2008). Aconceptual framework for improving the accessibility of fitness and recreation facilities for people with disabilities. Journal of Physical Activity and Health, 5, 158-168.

Rimmer, J., Padalabalanarayanan, S., Malone, L.A., \& Mehta, T. (2017). Fitness facilities still lack accessibility for people with disabilities. Disability and Health Journal, 10(2), 214-221. doi: 10.1016/j.dhjo.2016

Rimmer, J., Riley, B., Wang, E., \& Rauworth, A. (2004). Development and validation of AIMFREE: Accessibility instruments measuring fitness and recreation environments. Disability and Rehabilitation, 26(18), 1087-1095.

Rimmer, J., Riley, B., Wang, E., \& Rauworth, A., \& Jurkowski, J. (2005). Physical activity participation among persons with disabilities: barriers and facilitators. American Journal of Preventive Medicine, 26(5), 419-425.

Rimmer, J., Riley, B., Wang, E., \& Rauworth, A. (2005). Accessibility of health clubs for people with mobility disabilities and visual impairments. American Journal of Public Health, 95(11), 2022-2028.

Rimmer, J. A., \& Rowland, J. L. (2008). Physical activity for youth with disabilities: A critical need in an underserved population. Developmental Neurorehabilitation, 11(2), 141-148. doi: 10.1080/17518420701688649

Rodríguez de Luengo, M. R. (1999). Accesibilidad: Un objetivo prioritario para el siglo XXI. Integración, 30, 16-23.

Rowland, J. L, Fragala-Pinkham, M., Miles, C., \& O’Neil, M. E. (2015). The scope of pediatric physical therapy practice in health promotion and fitness for youth with disabilities. Pediatric Physical Therapy, 27(1), 2-15.

Stark, S., Hollingsworth, H. H., Morgan, K. A., \& Gray, D. B. (2007). Development of a measure of receptivity of the physical environment. Disability and Rehabilitation, 29(2), 123-137.

Thomas, J., \& Nelson, J. (2007). Métodos de investigación en actividad física. Barcelona: Paidotribo.

UNE 170001 (2007). Accesibilidad universal. Parte 1: Criterios DALCO para facilitar la accesibilidad al entorno. Madrid:AENOR.

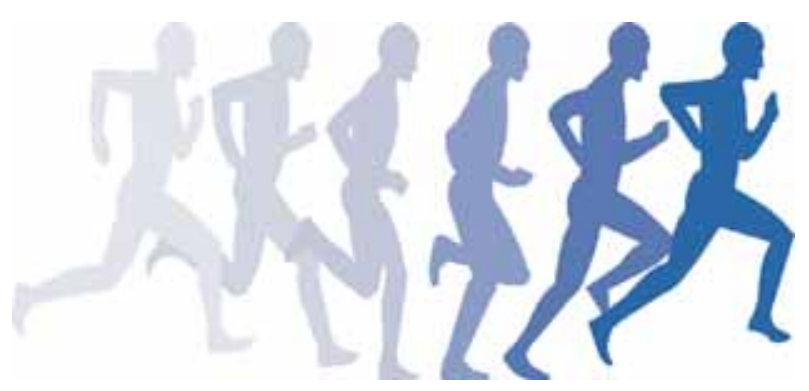

